\title{
Ocular surface symptoms among individuals exposed to ambient levels of traffic derived air pollution - a cross-
}

\section{sectional study [version 1; peer review: 2 approved with}

\section{reservations]}

\author{
Nabin Paudel (D), Sanjeev Adhikari, Sarina Manandhar, Ashesh Acharya, \\ Ajit Thakur, Bhairaja Shrestha
}

Drishti Eye Care Center, Kathmandu, 44620, Nepal

V1 First published: 21 Dec 2017, 6:2167

https://doi.org/10.12688/f1000research.13483.1

Latest published: 18 Sep 2018, 6:2167

https://doi.org/10.12688/f1000research.13483.2

\section{Abstract}

Background: The ocular surface is separated by a thin layer of tear film from outdoor air pollutants making individuals exposed to outdoor air pollution prone to various ocular surface disorders. The aim of this study was to determine the magnitude of ocular surface disorders symptoms among traffic police officers of Kathmandu, Nepal.

Methods: Two hundred traffic police officers working at different traffic police office branches of Kathmandu, Nepal were invited to the police headquarters for eye and vision examination. Among them, 91 individuals ( $95 \%$ males) completed the ocular surface disease index (OSDI) questionnaire and underwent Schirmer's I tear test.

Results: Symptoms of ocular surface disorders were reported by over $80 \%$ of the individuals. Approximately two fifths of the individuals (38\%) reported severe symptoms. Only $17 \%$ of the individuals' tear secretion was found to be below normal using the Schirmer's tear test. There was no association between the OSDI score and Schirmer's tear test scores $(r=0.008, p=0.94)$. A weak but significant relationship was observed between the OSDI score and job duration $(r=0.21, p=0.04)$. Individual exposed to outdoor air pollution for more than 10 years had higher odds of reporting ocular surface complaints as compared to those who were exposed for less than 10 years (OR = 3.94, $p=0.02$ ).

Conclusion: Ocular surface disorder symptoms are common among traffic police officers of Kathmandu, Nepal. The duration of exposure appears to significantly contribute to the increased symptoms in this

\section{Open Peer Review Approval Status \\ 1 2 \\ version 2 \\ (revision) \\ 18 Sep 2018 \\ version 1 \\ 21 Dec 2017

$\begin{array}{cc} & \\ \text { view } & \text { view } \\ ? & ? \\ \text { view } & \text { view }\end{array}$ \\ 1. Luc LR Int Panis (ID), Flemish Institute for Technological Research (VITO), Mol, Belgium Hasselt University, Hasselt, Belgium \\ 2. Monique Matsuda (D), University of São \\ Paulo, São Paulo, Brazil}

Any reports and responses or comments on the article can be found at the end of the article. 
vulnerable population.

Keywords

Air pollution, ocular surface, OSDI questionnaire, Kathmandu, Dry Eye

Corresponding author: Nabin Paudel (paudel.nabin@gmail.com)

Author roles: Paudel N: Conceptualization, Investigation, Methodology, Resources, Writing - Original Draft Preparation; Adhikari S: Investigation, Resources, Writing - Review \& Editing; Manandhar S: Investigation, Resources, Writing - Review \& Editing; Acharya A: Investigation, Resources, Writing - Review \& Editing; Thakur A: Investigation, Resources, Writing - Review \& Editing; Shrestha B: Methodology, Resources, Writing - Review \& Editing

Competing interests: No competing interests were disclosed.

Grant information: The author(s) declared that no grants were involved in supporting this work.

Copyright: $\odot 2017$ Paudel $\mathrm{N}$ et al. This is an open access article distributed under the terms of the Creative Commons Attribution License , which permits unrestricted use, distribution, and reproduction in any medium, provided the original work is properly cited. Data associated with the article are available under the terms of the Creative Commons Zero "No rights reserved" data waiver (CC0 1.0 Public domain dedication).

How to cite this article: Paudel N, Adhikari S, Manandhar S et al. Ocular surface symptoms among individuals exposed to ambient levels of traffic derived air pollution - a cross-sectional study [version 1; peer review: 2 approved with reservations] F1000Research 2017, 6:2167 https://doi.org/10.12688/f1000research.13483.1

First published: 21 Dec 2017, 6:2167 https://doi.org/10.12688/f1000research.13483.1 


\section{Introduction}

Studies conducted so far on air pollution and the human ocular surface have demonstrated a link between air pollution and ocular discomfort, abnormal tear structure, and ocular surface inflammation ${ }^{1}$. There are only a handful of studies demonstrating the association between the signs and symptoms of the ocular surface with air pollution ${ }^{2,3}$. Studies are even more infrequent from cities in developing countries, where the concentration of air pollutants in the environment is on the rise. Kathmandu is considered as one of the most highly polluted cities in the world, and Nepal is listed as one of the most polluted countries according to the WHO urban air pollution database. Traffic police officers in Kathmandu spend most of their time outdoors, controlling the flow of vehicles because of the unavailability of modern electronic traffic management systems in the city.

The purpose of this study was to determine the magnitude of ocular surface disorders based on a subjective symptoms questionnaire and a commonly used tear secretion test (Schirmer's I test), and then explore the association between these two tests in traffic police officers of Kathmandu, Nepal.

\section{Methods}

Study population

This study involved a cross-sectional, community-based assessment on 91 traffic police officers ( 86 male, 5 female) recruited among the officers of the Traffic Metropolitan head office, Baggikhana, Kathmandu, Nepal. The participants were invited by word of mouth by the head officer along with a formal written notice. Participants with any chronic illness, smoking habit, taking any systemic drugs, having any ocular diseases, previous ocular surgery and current contact lens wear were excluded from the study. All of the individuals had presenting visual acuity of better than 20/25 at both near and far. Only those participants who met our inclusion criteria and agreed to participate were included in the study. The study was conducted in the month of August 2017.

\section{Ethics and consent}

The study protocol was approved by the Ethics Committee of the Nepal Health Research Council (Reg.No.,218/2017). The study was part of a larger program that was aimed at determining ocular and visual disorders in police officers. All of the research participants provided their written informed consent for participation before being enrolled in the study. The Declaration of Helsinki was followed while assessing the participants.

\section{Ocular Surface Disease Index (OSDI) Questionnaire}

The Ocular Surface Disease Index Questionnaire ${ }^{4}$ is a validated tool to assess the subjective symptoms of individuals with potential ocular surface disorders. The Nepali translated OSDI questionnaire was administered to all of the participants before conducting the clinical assessment. An OSDI score of 0-12 was considered normal, 13-22 as mild, 23-32 as moderate, and $33-100$ as a severe ocular surface disorder ${ }^{5}$.

\section{Schirmer I test}

The Schirmer I tear test was conducted under topical anesthesia $(0.5 \%$ Proparacaine $)$. The test was conducted in an indoor setting at room temperature. After instilling one drop of proparacaine in each eye, the eye was dried with cotton for any residual drop. The Schirmer strip was then placed on the lateral $1 / 3 \mathrm{rd}$ aspect of the lower eye lid taking special care not to touch the cornea. The strip was removed from the lid after 5 minutes. The measurement of $5 \mathrm{~mm}$ or less was considered as abnormal.

\section{Ophthalmological examination}

Routine ophthalmological examination including visual acuity, refraction, anterior segment assessment and posterior segment assessment was also conducted but were not analysed as a part of this study

\section{Other variables}

Other variables such as age, gender, and duration of working as a traffic officer were also recorded.

\section{Statistical analysis}

The OSDI score was calculated using the following formula:

\section{(Sum of scores for all questions answered) X 25}

(total number of questions answered)

Data are presented as mean $\pm \mathrm{SD}$ unless mentioned otherwise. Independent sample t-test was employed for comparing mean between two groups whereas one way ANOVA, along with appropriate posthoc tests, was employed for comparing means between three or more groups. Pearson correlation was employed to determine the association between variables. Binary logistic regression analysis was also employed to determine association between dependent and independent variables. Statistical analysis was conducted using SPSS V22, IBM, California

\section{Results}

The mean age of the participants was $32 \pm 6$ years. The OSDI questionnaire was completed by all subjects. The mean OSDI score was $30.11 \pm 19.70$ (range 2 to 97.90). Based on the OSDI score, $81 \%$ of the participants reported symptoms of ocular surface disorder; over one third $(38 \%)$ of the participants reported symptoms of severe ocular surface disorder (Figure 1).

Schirmer's test of both eyes was conducted in $91 \%$ of the participants. The mean \pm SD Schirmer's test value $(\mathrm{mm})$ for right eye (RE) and left eye (LE) was $16.12 \pm 10.42$ and $17.42 \pm 10.84$, respectively. There was a high correlation $(\mathrm{r}=0.80, \mathrm{p}<0.001)$ but a non-significant difference $(\mathrm{p}=0.08)$ in the Schirmer's test score between the two eyes. Hence the results of the right eye only were used for analysis. Only $17 \%$ of the subjects' Schirmer's score showed abnormal results. 
No association was observed between the OSDI score and the Schirmer's test results $(r=0.008, p=0.94)$. No significant correlation was also observed between the OSDI scores and age $(\mathrm{r}=0.15, \mathrm{p}=0.14)$. A weak, but statistically significant, positive correlation was observed between OSDI score and duration of work $(\mathrm{r}=0.21, \mathrm{p}=0.04)$. The mean duration of work was $11 \pm 6$ years. Individuals who had held the job for more than 5 years had severe symptoms, as compared to those who had held the job for less than five years $(p=0.001)$. A one way ANOVA test demonstrated a significant difference in the OSDI score between different age groups $(<30,30-40$ and $>40$ years) $\left(\mathrm{F}_{2.88}=3.86, \mathrm{p}=0.025\right)$. The symptoms score was statistically significantly different between individuals who had worked for up to 5 years, six to ten years (mean difference, $13.65 \pm 6.44$, $95 \%$ CI, 0.85 to 26.46 ) and more than 10 years (mean difference,
$16.48 \pm 5.93,95 \%$ CI, 4.70 to 28.27 ). However, no statistically significant difference was observed between individuals who had held the job for 6-10 years and $>10$ years (mean difference $2.82 \pm 4.54,95 \%$ CI, -6.20 to 11.50 ) (Figure 2). Furthermore, individuals who held the job for 10 years or more had significantly higher odds of having ocular surface symptoms as compared to those who had the job for less than ten years (OR: 3.94, 95\% CI, 1.25-12.8, p = 0.02). There was a slight increase in the odds of having ocular surface symptoms after adjusting for age and gender, but it was borderline significant (OR: 4.28, 95\% CI, 0.93-19.58, $\mathrm{p}=0.05$ )

Of the 74 subjects identified as having symptoms of ocular surface disorders according to the OSDI score, only 16 were identified as abnormal by the Schirmer's test.

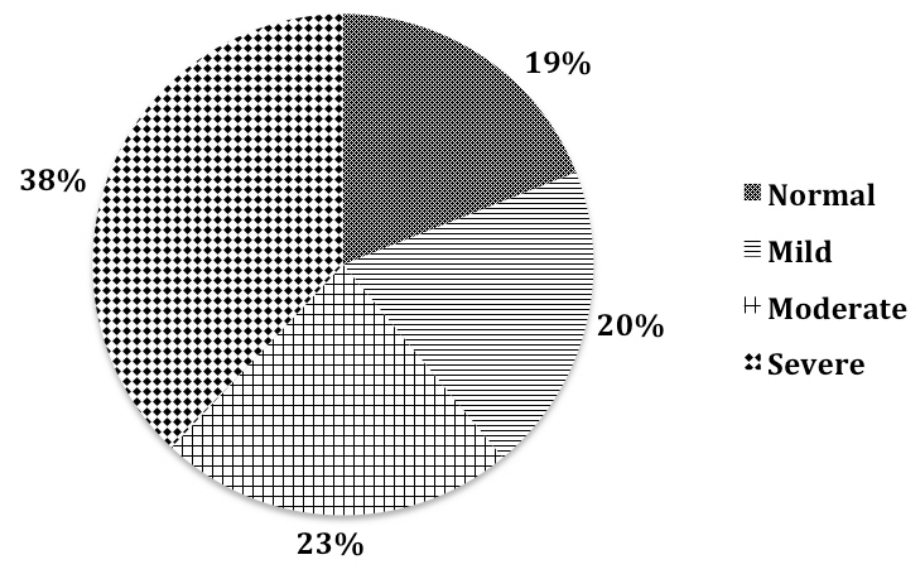

Figure 1. Frequency of ocular surface disorder symptoms according to the OSDI score.

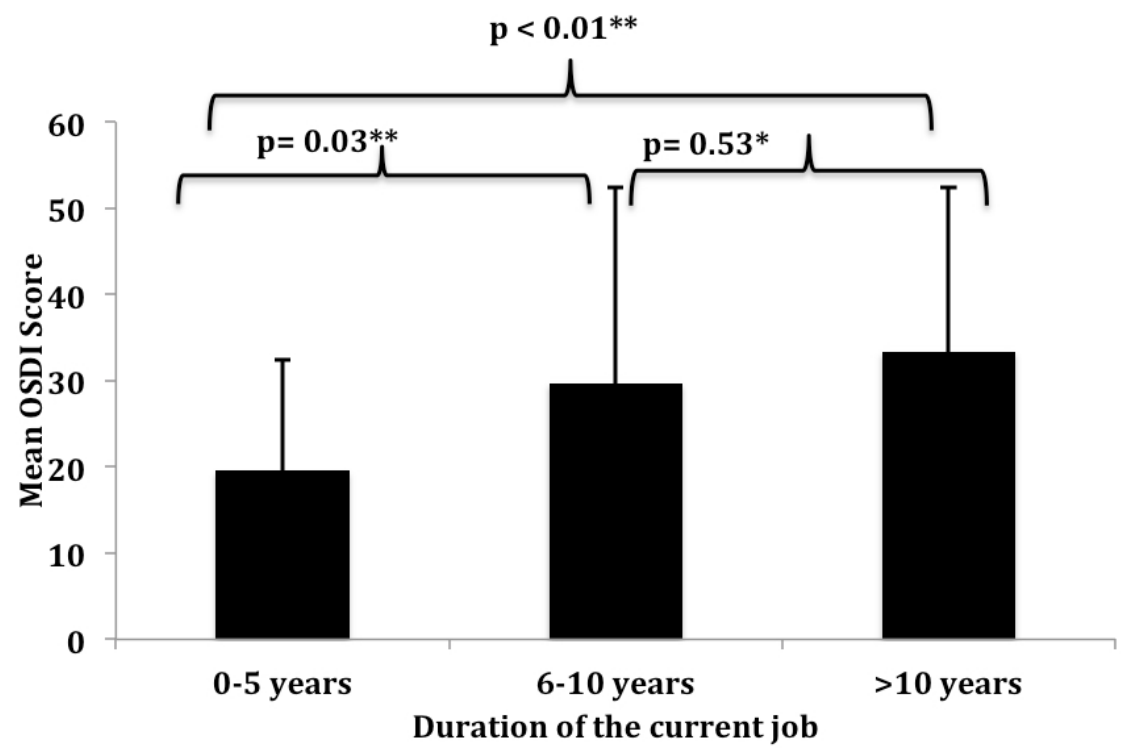

Figure 2. Variation of the OSDI score according to the duration of the job. ${ }^{* *}=$ statistically significant, ${ }^{*}=$ non-significant). Error bars denote standard deviation. 
Dataset 1. Data on the ocular surface symptoms among individuals exposed to ambient levels of air pollution

http://dx.doi.org/10.5256/f1000research.13483.d188591

\section{Discussion}

This study explored the symptoms of ocular surface disorders among individuals exposed to traffic-derived air pollution in Kathmandu, Nepal. A remarkable number of individuals reported symptoms with over one third reporting symptoms of severe ocular surface disorder. Ocular surface disorder vary with age, whereby the prevalence is $11 \%$ among individuals between 40 to 59 , and $18 \%$ in individuals above $80^{6}$. In this study, individuals were between 18 to 48 years, and $80 \%$ had symptoms of OSD, which is alarmingly high as compared to the general population $^{6}$.

Previous reports exploring symptoms in individuals exposed to traffic-derived air pollution have found mixed results. The Torricelli et al. ${ }^{7}$ study in a group of 71 taxi drivers and traffic controllers reported that most of their subjects reported few symptoms, and fell within the normal category according to the OSDI scoring. However, they demonstrated that objective tests such as tear osmolarity and break up time were significantly reduced. In contrast, Saxena et al. reported that most of the subjects who were exposed to air pollution had more symptoms (irritation, itching, lacrimation, and redness) as compared those who were not exposed ${ }^{2}$.

A majority of the individuals' Schirmer's results were within normal range in the present study. Similar normal findings of Schirmer's test have been found by previous researchers ${ }^{7,8}$. This finding is not surprising as the poor diagnostic ability of the Schirmer's test for detecting ocular surface dysfunction has been well recorded in the literature?. The Schirmer's test has shown normal results in many previous studies conducted among established dry eye population ${ }^{9}$.

The lack of correlation between the OSDI scores and the Schirmer's results is also not surprising, as this finding is consistent with most of the previous studies where the signs and symptoms of ocular surface disorders, particularly that of the dry eyes, are not correlated with one another ${ }^{10}$. It is postulated that dry eye is a multifactorial disorder, and different mechanisms and factors act in compliment or may act independently to elicit the symptomatology of this condition ${ }^{11}$.

The weak but statistically significant positive correlation between the OSDI score and duration of holding the current job (years) implies that the longer the exposure, the more severe the symptoms. However, the finding that the mean symptom score is not significantly different between individuals who have held the job for 6-10 years and in those over 10 years signifies that exposure to ambient air pollution over 5 years poses a significant impact on the ocular surface. Furthermore, the higher odds of having ocular symptoms in individuals with over 10 years of holding the job implies that the effect of air pollution on the ocular surface may have a cumulative effect over the years until symptoms start to appear.

Nepal was ranked as the 177th country just above China, Bangladesh, and India among the 180 countries with air quality issues according to the Environmental Performance Index (EPI) of $2016^{12}$. A report in 2007 on the air pollution concentration, specifically of the $\mathrm{PM}_{2.5}$ of the Kathmandu valley, was found to be $17-18$ fold higher than the recommended $25 \mathrm{ug} / \mathrm{m}^{3}$ threshold provided by the WHO. A 2016 air pollution report of Nepal provided by the WHO has shown a considerable increase in $\mathrm{PM}_{25}$ concentration over a decade ${ }^{13}$. Our study was conducted in the month of August 2017. The mean 24-hour average $\mathrm{PM}_{2.5}$ concentration during that month was $113.5 \mathrm{ug} / \mathrm{m}^{3}$ (approximately 5 fold higher than that recommended by the WHO) and the $\mathrm{PM}_{10}$ concentration was $633 \mathrm{ug} / \mathrm{m}^{3}$ (approximately 13 fold higher than the WHO recommendation) (see Kathmandu Air Pollution: Realtime Air Quality Index and Department of Environment, Air Quality Monitoring). In light of the high levels of air pollution of Kathmandu, the higher number of individuals reporting severe symptoms of ocular surface disease in our study can be explained.

While this study provided novel ocular health issues in this vulnerable population, some limitations must be acknowledged. Firstly, only two tests were used - the OSDI questionnaire and the Schirmer's I test to determine ocular surface disorder. Use of more sensitive tests such as corneal and conjunctival staining, tear film break up time and tear osmolarity would have detected more individuals with ocular surface disorders, and may also have demonstrated structural/physiological anomalies of the ocular surface. However, as this was a community-based study, tests were chosen based on the non-requirement of sophisticated clinical instruments and investigations. Secondly, the actual duration and concentration of air pollution exposures in our subjects were not assessed. Measurement of the $\mathrm{PM}_{25}$ and $\mathrm{NO}_{2}$ concentration, along with a range of ocular surface disorder diagnostic tests like that of a few previous studies, would have provided us a better understanding of the association between air pollution and ocular surface disorders. Thirdly, a comparison with a control group of individuals who were not exposed to a different level of air pollution would have confirmed that the ocular symptoms were primarily due to air pollution. Nevertheless, this study was a first step toward generating awareness, and exploring symptoms related to ocular surface disorder in the vulnerable population. Future large-scale studies need to be conducted in city areas to explore ocular surface anomalies in this vulnerable population and necessary precautions are taken in order to protect the ocular health of people exposed to outdoor air pollution.

\section{Conclusion}

Traffic police officers of Kathmandu valley have a high prevalence of ocular surface complaints, which do not correlate well with the subjective tear secretion test. The duration of job appears to somewhat contribute to the increasing symptoms. In the meantime, the use of protective sunglasses and regular eye consultations for people who are exposed to outdoor air pollution is 
recommended. More importantly, the government must implement new rules to reduce the levels of outdoor air pollution.

\section{Data availability}

Dataset 1: Data on the ocular surface symptoms among individuals exposed to ambient levels of air pollution. DOI: 10.5256/f1000research.13483.d188591 ${ }^{12}$
Competing interests

No competing interests were disclosed.

\section{Grant information}

The author(s) declared that no grants were involved in supporting this work.
1. Torricelli $A A$, Novaes $P$, Matsuda $M$, et al: Ocular surface adverse effects of ambient levels of air pollution. Arq Bras Oftalmol. 2011; 74(5): 377-81. PubMed Abstract | Publisher Full Text

2. Saxena $R$, Srivastava $S$, Trivedi $D$, et al:: Impact of environmental pollution on the eye. ActaOphthalmologica. 2003; 81(5): 491-4.

PubMed Abstract | Publisher Full Text

3. Versura $\mathrm{P}$, Profazio V, Cellini M, et al.: Eye discomfort and air pollution Ophthalmologica. 1999; 213(2): 103-9. PubMed Abstract | Publisher Full Text

4. Miller KL, Walt JG, Mink DR, et al:: Minimal Clinically Important Difference for the Ocular Surface Disease Index. Arch Ophthalmol. 2010; 128(1): 94-101. PubMed Abstract | Publisher Full Text

5. Moss SE, Klein R, Klein BE: Prevalence of and risk factors for dry eye syndrome. Arch Ophthalmol. 2000; 118(9): 1264-1268. PubMed Abstract | Publisher Full Text

6. Torricelli AA, Novaes $\mathrm{P}$, Matsuda M, et al:: Correlation between signs and symptoms of ocular surface dysfunction and tear osmolarity with ambient levels of air pollution in a large metropolitan area. Cornea. 2013; 32(4): e11-5.

PubMed Abstract | Publisher Full Text

7. Novaes $\mathrm{P}$, Saldiva $\mathrm{PH}$, Matsuda M, et al:: The effects of chronic exposure to traffic derived air pollution on the ocular surface. Environ Res. 2010; 110(4): $372-4$

PubMed Abstract | Publisher Full Text

8. Cho P, Yap M: Schirmer test. I. A review. Optom Vis Sci. 1992; 70(2): 152-6. PubMed Abstract | Publisher Full Text

9. Nichols K, Nichols J, Mitchell G: The lack of association between signs and symptoms in patients with dry eye disease. Cornea. 2004; 23(8): 762-770. PubMed Abstract | Publisher Full Text

10. Lemp MA: Advances in Understanding and Managing Dry Eye Disease. Am J Ophthalmol. 2008; 146(3): 350-356.

PubMed Abstract | Publisher Full Text

11. Hsu A, Zomer A: Environmental Performance Index. Wiley StatsRef: Statistics Reference Online. 2016; 1-5. Publisher Full Text

12. Paudel N, Adhikari S, Manandhar S, et al: Dataset 1 in: Ocular surface symptoms among individuals exposed to ambient levels of traffic derived air pollution - a cross-sectional study. F1000Research. 2017. Data Source

13. Schiffman $\mathrm{R}$, Christianson M: Reliability and validity of the ocular surface disease index. ArchOphthalmol. 2000; 118(5): 615-21.

PubMed Abstract | Publisher Full Text 


\section{Open Peer Review}

\section{Current Peer Review Status: ? ?}

\section{Version 1}

Reviewer Report 20 August 2018

https://doi.org/10.5256/f1000research.14641.r35769

(C) 2018 Matsuda M. This is an open access peer review report distributed under the terms of the Creative Commons Attribution License, which permits unrestricted use, distribution, and reproduction in any medium, provided the original work is properly cited.

\section{Monique Matsuda}

Laboratory of Ophthalmology, School of Medicine, University of São Paulo, São Paulo, Brazil

The study evaluated the eye symptoms and the lacrymal production of traffic police officers in Kathmandu, Nepal, a city with high levels of air pollution.

As the study refers to the effects of air pollution on the ocular surface of traffic police officers, it is necessary to mention the average concentration of air pollutants during August 2017, mainly PM 2.5 and $\mathrm{NO}_{2}$. Vehicles are generally the main responsible for the emission of nitrogen oxides, since in Kathmandu vehicular traffic seems to be very intense, the additional information about these air pollutants is essential as part of the study.

In addition to the air pollutants, I suggest mentioning the meteorological data, such as humidity and temperature, since these climatic factors may influence the clinical parameters of the ocular surface.

The study was conducted during the hot and rainy season. In this period there is an increase of allergies and conjunctivitis and among the symptoms, an increased frequency of itching, foreign body sensation and photophobia. In addition, the use of fans and air conditioning is greater. All these factors could influence and favor the appearance of symptoms and the increase of OSDI score. Thus, in future studies, it would be interesting to carry out the same tests and ophthalmologic examinations during the dry season in the same group of traffic police officers.

Besides that, OSDI checks the symptoms during the last week. The reproducibility of symptoms of the OSDI questionnaire at different periods could be indicative of the prevalence of symptoms for a long period due to air pollution exposure and could be more certainty correlate with the working time in the traffic. I suggest that the correlation between the OSDI score and working time should be mentioned with caution in the text, once this is a cross-sectional study.

I recommend the availability of an OSDI table describing the frequency of symptoms in the attachment. 
The authors mentioned very well the limitations of the study. Despite the application of only two ophthalmological parameters (OSDI questionnaire and Schirmer test), I recommend the indexing of the article since studies about the effects of air pollution in areas of high air pollutant levels, as in Kathmandu, it is necessary to evidence its effects on the ocular surface and to strengthen public policies in this area.

I suggest minor language revision.

Is the work clearly and accurately presented and does it cite the current literature? Partly

Is the study design appropriate and is the work technically sound?

Partly

Are sufficient details of methods and analysis provided to allow replication by others? Yes

If applicable, is the statistical analysis and its interpretation appropriate?

Yes

Are all the source data underlying the results available to ensure full reproducibility? Partly

Are the conclusions drawn adequately supported by the results?

Yes

Competing Interests: No competing interests were disclosed.

Reviewer Expertise: Air pollution and effects on the ocular surface

I confirm that I have read this submission and believe that I have an appropriate level of expertise to confirm that it is of an acceptable scientific standard, however I have significant reservations, as outlined above.

Author Response 11 Sep 2018

Nabin Paudel, Drishti Eye Care Center, Kathmandu, Nepal

Dear Dr Matsuda,

Thank you for your time to review our manuscript and providing us with an opportunity to revise the manuscript. We have endeavoured to address each comment and suggestions. Please see below.

Comment: "As the study refers to the effects of air pollution on the ocular surface of traffic police officers, it is necessary to mention the average concentration of air pollutants during August 2017, mainly $\mathrm{PM}_{2.5}$ and $\mathrm{NO}_{2}$. Vehicles are generally the main responsible for 
the emission of nitrogen oxides, since in Kathmandu vehicular traffic seems to be very intense, the additional information about these air pollutants is essential as part of the study."

Response: Thank you very much for your comment. We agree with the comments. We had already provided the August 2017 data on $\mathrm{PM}_{2.5}$ and $\mathrm{PM}_{10}$ concentration in the first version. As there is no national mechanism to measure the $\mathrm{NO}_{2}$ system in Nepal the data from August 2017 is not available. However, in our latest version, we have included the $\mathrm{NO}_{2}$ data of 2015. Please refer to the introduction.

Comment: In addition to the air pollutants, I suggest mentioning the meteorological data, such as humidity and temperature, since these climatic factors may influence the clinical parameters of the ocular surface.

Response: Thank you. The meteorological data has now been added.

Comment: The study was conducted during the hot and rainy season. In this period there is an increase of allergies and conjunctivitis and among the symptoms, an increased frequency of itching, foreign body sensation and photophobia. In addition, the use of fans and air conditioning is greater. All these factors could influence and favor the appearance of symptoms and the increase of OSDI score. Thus, in future studies, it would be interesting to carry out the same tests and ophthalmologic examinations during the dry season in the same group of traffic police officers.

Response: We absolutely agree with the comment. We have included these factors in the discussion. We also agree that it would be interesting to carry out the same tests and ophthalmologic examination during the dry season in the same group to gain a better understanding of the causative factors of ocular symptoms in this population. We are planning such a study at the moment.

Comment: Besides that, OSDI checks the symptoms during the last week. The reproducibility of symptoms of the OSDI questionnaire at different periods could be indicative of the prevalence of symptoms for a long period due to air pollution exposure and could be more certainty correlate with the working time in the traffic. I suggest that the correlation between the OSDI score and working time should be mentioned with caution in the text, once this is a cross-sectional study.

Response: Thank you very much for this close observation. We have mentioned the crosssectional nature of the study as one of the limitations and hence have advised that caution must be applied before interpreting the research findings.

Comment: I recommend the availability of an OSDI table describing the frequency of symptoms in the attachment.

Response: We have attached the OSDI form as an attachment. 
Comment: The authors mentioned very well the limitations of the study. Despite the application of only two ophthalmological parameters (OSDI questionnaire and Schirmer test), I recommend the indexing of the article since studies about the effects of air pollution in areas of high air pollutant levels, as in Kathmandu, it is necessary to evidence its effects on the ocular surface and to strengthen public policies in this area.

Response: We are thankful for your kind comments.

Competing Interests: No competing interests.

Reviewer Report 22 June 2018

https://doi.org/10.5256/f1000research.14641.r31452

(C) 2018 Int Panis L. This is an open access peer review report distributed under the terms of the Creative Commons Attribution License, which permits unrestricted use, distribution, and reproduction in any medium, provided the original work is properly cited.

\section{Luc LR Int Panis}

${ }^{1}$ Environmental Risk and Health, Flemish Institute for Technological Research (VITO), Mol, Belgium

2 Transportation Research Institute, Hasselt University, Hasselt, Belgium

This paper describes a limited analysis of eye symptoms in a small group of traffic police officers in Kathmandu, Nepal. Despite the limitations of the cross-sectional set-up and limited resources, this analysis merits publication because of the small volume of studies on ophthalmological effects of air pollution and the health effects of air pollution in non-western countries with extremely high air pollution exposures.

The article would benefit from a brief description of concentrations of other common (gaseous or solid) air pollutants (NO2, CO, BC) during August 2017 and annual averages in the previous years in Kathmandu if available.

The total number of questions in the OSDI questionnaire could be mentioned to facilitate the interpretation of the formula used.

The description of the Schirmer I test is too brief to be easily understood by air pollution scientists who are no experts in ophthalmology. To consider only values $<5 \mathrm{~mm}$ as abnormal seems to be a very strict definition e.g. compared to results presented by Karampatakis et al. ${ }^{1}$. Based on RE \& LE combined the $\%$ of abnormal tests is less than $15 \%$ (not $17 \%$ ).

Also there is no explanation about the OSDI of the $9 \%$ of participants that did not undergo a Schirmer I test, this could potentially lead to biased results.

In Figure 1 the categories of Mild (23\%) and Moderate (20\%) seem to have been mixed up. The authors should double check whether this also happened with other categorizations (not available from the provided data) and repeat the statistical analysis if necessary. By providing more original 
(not categorized) data reanalysis of the data by other researchers would also be facilitated. The methods section does not mention that a paired t-test was used to compare results of the Schirmers I test for RE and LE.

Because of the cross-sectional set-up of the study it would be more prudent to avoid the word 'implies' in the discussion and instead use 'suggests'.

The authors provide a good assessment of some of the limitations/weaknesses of their study. Including the lack of a control group, lack of personal/detailed exposure data or on groups with a different level of exposure and lack of more sophisticated eye tests. The inherent limitations of the cross-sectional set-up should also be mentioned.

The study population of traffic police officers could perhaps better be characterized as a 'more exposed' population instead of 'vulnerable' unless there would be a reason why these adults would be more vulnerable than other population groups.

With respect to their recommendation of wearing sunglasses the authors should provide a reference to a source providing evidence for the benefits of such an intervention. Were the traffic police officers questioned on the frequency of use of sunglasses during work?

Some minor language issues could be corrected.

\section{References}

1. Karampatakis V, Karamitsos A, Skriapa A, Pastiadis G: Comparison between normal values of 2and 5-minute Schirmer test without anesthesia.Cornea. 2010; 29 (5): 497-501 PubMed Abstract | Publisher Full Text

Is the work clearly and accurately presented and does it cite the current literature? Partly

Is the study design appropriate and is the work technically sound? Partly

Are sufficient details of methods and analysis provided to allow replication by others? Partly

If applicable, is the statistical analysis and its interpretation appropriate? Partly

Are all the source data underlying the results available to ensure full reproducibility? Partly

Are the conclusions drawn adequately supported by the results? Yes

Competing Interests: No competing interests were disclosed. 
Reviewer Expertise: Traffic related air pollution and health

I confirm that I have read this submission and believe that I have an appropriate level of expertise to confirm that it is of an acceptable scientific standard, however I have significant reservations, as outlined above.

Author Response 11 Sep 2018

Nabin Paudel, Drishti Eye Care Center, Kathmandu, Nepal

Dear Dr Panis,

Thank you very much for taking the time to review our manuscript. We have done our best to address your comments. We hope that our reply is satisfactory and that we have responded to, and dealt with, all comments adequately. Please see responses below:

Comment: This paper describes a limited analysis of eye symptoms in a small group of traffic police officers in Kathmandu, Nepal. Despite the limitations of the cross-sectional set-up and limited resources, this analysis merits publication because of the small volume of studies on ophthalmological effects of air pollution and the health effects of air pollution in non-western countries with extremely high air pollution exposures.

Response: Thank you very much for your kind comments. We agree with the limitations of the study but hope that this study will be the first one to raise awareness among affected individuals and concerned parties regarding the effect of air pollution on ocular health.

Comment: The article would benefit from a brief description of concentrations of other common (gaseous or solid) air pollutants (NO2, CO, BC) during August 2017 and annual averages in the previous years in Kathmandu if available.

Response: Thank you very much. We apologise for the unavailability of the $\mathrm{NO}_{2}, \mathrm{CO}$ and $\mathrm{BC}$ data for the month of August 2017 as Nepal does not have a national mechanism to collect these data. We have gathered as much relevant information as we can from the published literature and have incorporated in the latest version.

Comment: The total number of questions in the OSDI questionnaire could be mentioned to facilitate the interpretation of the formula used.

Response: This has now been incorporated with the OSDI questionnaire attached as a supplementary file.

Comment: The description of the Schirmer I test is too brief to be easily understood by air pollution scientists who are no experts in ophthalmology. To consider only values $<5 \mathrm{~mm}$ as abnormal seems to be a very strict definition e.g. compared to results presented by Karampatakis et al. ${ }^{1}$

Response: Thank you for this thoughtful comment. We have included some additional information regarding the Schirmer's test. The value of $<5 \mathrm{~mm}$ for abnormal was based on 
its diagnostic accuracy. This has been briefly mentioned in the manuscript.

Comment: Based on RE \& LE combined the \% of abnormal tests is less than 15\% (not 17\%). Also there is no explanation about the OSDI of the $9 \%$ of participants that did not undergo a Schirmer I test, this could potentially lead to biased results.

Response: Thank you for pointing this out. We have rectified the percentage. We apologise for the lack of clarification regarding the $91 \%$ who underwent the Schirmer Test. This data was based on an earlier data analysis. We did not include the data from those patients who had incomplete information hence they were not analysed in this study. Only participants who had both the Schirmer's and OSDI scores were included in the study.

Comment:In Figure 1 the categories of Mild (23\%) and Moderate (20\%) seem to have been mixed up. The authors should double check whether this also happened with other categorizations (not available from the provided data) and repeat the statistical analysis if necessary. By providing more original (not categorized) data reanalysis of the data by other researchers would also be facilitated.

Response: We have now rectified the percentage. This was an error during plotting the graph. We confirm that this has not affected any other analysis. The dataset file consists of all the information that we collected and were relevant to the study project. The categorisation of age group was suggested by the editorial office.

Comment: The methods section does not mention that a paired t-test was used to compare results of the Schirmers I test for RE and LE.

Response: Thank you very much. We have mentioned it now.

Comments: Because of the cross-sectional set-up of the study it would be more prudent to avoid the word 'implies' in the discussion and instead use 'suggests'.

Response: We have changed to suggests as recommended.

Comments: The authors provide a good assessment of some of the limitations/weaknesses of their study. Including the lack of a control group, lack of personal/detailed exposure data or on groups with a different level of exposure and lack of more sophisticated eye tests. The inherent limitations of the cross-sectional set-up should also be mentioned.

Response: Thank you so much for your kind comments. We have added the cross-sectional nature of the study as one of the limitations and have advised readers that the results must be interpreted with caution.

Comments: The study population of traffic police officers could perhaps better be characterized as a 'more exposed' population instead of 'vulnerable' unless there would be a reason why these adults would be more vulnerable than other population groups. 
Response: We have changed vulnerable to more exposed. Thank you.

Comment: With respect to their recommendation of wearing sunglasses the authors should provide a reference to a source providing evidence for the benefits of such an intervention. Were the traffic police officers questioned on the frequency of use of sunglasses during work?

Response: We have added a reference that reports the beneficial effect of glasses on ocular surface disorders such as dry eyes. Unfortunately, we did not ask any questions regarding the frequency of the use of sunglasses during work but will definitely consider for future studies.

Comments: Some minor language issues could be corrected.

Response: We have attempted to reduce such issues as much as possible.

Competing Interests: No competing interests.

The benefits of publishing with F1000Research:

- Your article is published within days, with no editorial bias

- You can publish traditional articles, null/negative results, case reports, data notes and more

- The peer review process is transparent and collaborative

- Your article is indexed in PubMed after passing peer review

- Dedicated customer support at every stage

For pre-submission enquiries, contact research@f1000.com 